\title{
Formulation And Characterization of Carageenan Gels Encapsulating Amphotericin B And Lactobacillus acidophilus Against Candidal Vaginitis
}

\author{
R KAUR $^{1}$, M KHURANA $^{1}$, M BINDAL $^{1}$ AND A SHARMA ${ }^{1 *}$ \\ ${ }^{1}$ Department of Bioinformatics and Biotechnology, DAV, College, Sec-10, \\ Chandigarh-160017
}

*Email:anjs108@yahoo.co.in

Received: July 23, 2017l Revised: September 18, 2017l Accepted: September 26, 2017

Published online: October 02, 2017

The Author(s) 2017. This article is published with open access at www.chitkara.edu.in/ Publications

\begin{abstract}
The present study is concerned with the development and characterization of bioadhesive carageenan gel encapsulating Amphotericin B and Lactobacillus acidophillus, prepared by graft co-polymeriztion against Candidal vaginitis. Intravaginal gel systems based on bioadhesive polymer (carrageenan) were characterized with respect to swelling index, bioadhesive strength, percent encapsulation and in vitro drug release antimicrobial studies. A marked increase in swelling index of gel encapsulating Lactobacillus was found to be $1.9 \pm 0.35$. The percent encapsulation of drug was found to be $98.63 \% \pm .0 .2 \%$ and that of Lactobacillus was $91.81 \pm 0.01$. The viability was observed for interval of 6 hrs on trypton soya agar and showed that viability was highly conserved till $4 \mathrm{hrs}$. The antimicrobial study of gels encapsulating Amphotericin B and Lactobacillus showed that carageenan gel can inhibit Candida albicans upto a maximum extent. Bioadhesivity study also conducted for gels that showed a bioadhesivity of $84.66 \% \pm .0 .5 \%$ with drug, $88.66 \% \pm .02 \%$ with Lactobacillus. In-vitro drug release showed a sustained type release of drug from the polymer i.e. there was initial burst of Amphotericin B up to 5 hours, after which there was a sustained release upto 10 days. Thus it has been concluded from the present study that bioadhesive gels encapsulating Amphotericin B can act as promising drug carriers along with Lactobacillus against candidal vaginitis.
\end{abstract}

Keywords: Candidal Vaginitis, Carageenan, Swelling index, Encapsulation, Bioadhesivity

Journal of Multidisciplinary Research in Healthcare Vol-4, No-1, October 2017 pp. $37-49$ 
Kaur, R

Khurana, M

Bindal, $\mathrm{M}$

Sharma, A

\section{INTRODUCTION}

Candidal Vaginitis (candidiasis) is a typical problem caused by Candida albicans which is characterized by inflammation of the female genitalia. Vaginitis is very common and is reported by as many as $75 \%$ of women at some point in their lives. It is one of the most common forms of Candida infection in women. These fungi can be found all over the body and are normally present in moist areas of body. Donati and Di Vico (2010) has reported that 20\%$50 \%$ of all women normally carry yeast in the vagina without the presence of symptoms [1].

The vaginal flora is the dynamic system mainly consisting of Lactobacillus (Doderlein's bacilli) which is the most prevalent organism in vaginal environment together with many other facultative and obligate aerobes and anaerobes (Cornebacteria and yeast). The Lactobacilli produce sufficient lactic acid to acidify vaginal secretions to a $\mathrm{pH} 3.5-4.5$. An imbalance in the intestinal flora where good bacteria are killed due to excessive use of antibiotics, unhealthy lifestyles and a weak immune system usually cause over growth of yeast. Various drugs which are used to cure candidal vaginitis are Amphotericin B, Fluconazole, Nystatin, metronidazole, Itraconazole, clotrimazole etc. Traditionally solutions, suppositories, foams and tablets have been used as vaginal formulations. Amphotericin B is one of the effective drugs used against candidal vaginitis which is a macrocyclic polyene with antifungal activity. It is associated with ergosterol, forming a transmembrane channel that leads to monovalent ion leakage causing fungal death. The drugs has various side effects like irritation, diarrhoea, headache, hypersensitivity, low availability of drug, poor patient compliance, and systemic side effects due to higher total dose and high frequency of drug administration. Sustained release drug delivery systems offer a promising approach in overcoming these drawbacks The use of mucoadhesive polymers for development of gels may help in controlled release of bioactive agents. Hydrogels are hydrophilic polymer networks which have the capability to imbibe water or biological fluids. They can exhibit dramatic changes in their swelling behaviour, network structure, permeability and mechanical strength in response to change in $\mathrm{pH}$ or ionic strength of surrounding fluid or temperature Gupta (2012) [2]. Probiotics offer alternative anticandidal approach as they lower the $\mathrm{pH}$ and help in restoring the balance of microflora. Probiotics are living micro organisms that favourably influence the health of the host by improving the indigenous microflora. Boskey et al. (2001) has confirmed that Lactobacilli pl ay an important role in the inhibition of growth, adhesion and spread of pathogenic microbes and thus possess good antimicrobial properties [3] The present study is an endeavour to explore formulation and characterization of carageenan 
based hydrogels encapsulating amphotericin B and probiotics as effective drug delivery systems against Candidal vaginitis.

\section{MATERIALS AND METHODS}

Commercially available IP injection of "Amphotericin B" under the brand name of Fungitericin manufactured by Gufic biosciences limited,Kabilpore, Navsari, Gujarat state.(India) was used, Sporolac manufactured by Uni Sankyo Ltd. containing around 150 million spores of Lactobacillus sporogenes in $1 \mathrm{~g}$ sachet was used in the study, Carrageenan, tryptone soya agar, dialysis membrane manufactured by HiMedia was used in the study, Lyophilized culture of Candida albicans (MTCC-183) was procured from IMTECH, Chandigarh, India. All the reagents of standard grade were used in the study.

\subsection{Preparation of Carrageenan Based Vaginal Gel Hosseinzadeh (2010)[4]}

$1 \mathrm{~g}$ of kappa-carrageenan was dispersed in $30 \mathrm{ml}$ of distilled water. The dispersion was mixed using a magnetic stirrer for 20 minutes and then placed in a water bath at $80{ }^{\circ} \mathrm{C}$ for 20 minutes. After dissolving $\mathrm{K}$ - carrageenan, the initiator Ammonium per sulphate $(0.01 \mathrm{~g}$ in $5 \mathrm{ml}$ of distilled water) and Methylene bis acrylamide $(0.05 \mathrm{~g}$ in $5 \mathrm{ml}$ of distilled water) were simultaneously added. The mixture was again stirred on a magnetic stirrer for $20 \mathrm{~min}$. The monomer acrylamide $(1.0 \mathrm{~g})$ was added and gelation was observed after $15 \mathrm{~min} .1 \mathrm{ml}$ of the drug Amphotericin B and 500mg spores of Lactobacillus was added to the $10 \mathrm{ml}$ of gel and mixed thoroughly (By graft co-polymerisation ).

\subsection{Physicochemical Characterization of Gels EncapsulatingAmphotericin B and Lactobacillus}

\subsubsection{Determination of $\mathrm{pH}$ of vaginal gel}

$1 \mathrm{~g}$ of gel was dissolved in $30 \mathrm{ml}$ of distilled water and the electrode was then dipped in to gel formulation and constant reading was noted. The measurements of $\mathrm{pH}$ of each formulation were repeated three times Ramadan (2008) [5].

\subsubsection{Swelling Index Studies}

Swelling index is the measure of intensity of the gelation of the gel. More the swelling index, tighter the gel is and vice-versa.1g of gel was taken on a filter paper and then placed in a beaker containing SVF for 6 hours. Samples were removed from beakers at different time intervals $(30 \mathrm{~min}$., $60 \mathrm{~min}, 180 \mathrm{~min}$, $360 \mathrm{~min}$.) Samples were put in a dry place for some time after that these were reweighed at different time intervals as optimized by Pal and Nayak (2011) [6].
Formulation And

Characterization of Carageenan

Gels Encapsulating

Amphotericin B

And Lactobacillus acidophilus Against

Candidal Vaginitis

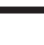


Kaur, R

Khurana, M

Bindal, $\mathrm{M}$

Sharma, A

Swelling Index was calculated using:

$\mathrm{Sw} \%=\left[\left(\mathrm{W}_{\mathrm{t}}-\mathrm{W}_{0}\right) / \mathrm{W}_{0}\right] \mathrm{X} 100$

$\mathrm{Sw}=$ Equilibrium percent swelling

$\mathrm{W}_{\mathrm{t}}=$ weight of swollen gel after time ' $\mathrm{t}$ '

$\mathrm{W}_{0}=$ original weight of gel at zero time.

Where, $\mathrm{T}=$ amount of total drug, $\mathrm{C}=\mathrm{drug}$ present in supernatant

\subsubsection{Determination of encapsulation efficiency of gel}

To $1 \mathrm{~g}$ of each vaginal gel $10 \mathrm{ml}$ of methanol was added in a volumetric flask followed by continuous shaking for 10 minutes and then diluted to $100 \mathrm{ml}$ with phosphate buffer $(\mathrm{pH}=7$.4and centrifuged. The supernatant was filtered. The absorbance of filtrate was taken at $416 \mathrm{~nm}^{5}$.

Percent Encapsulation $=\frac{\text { Totalamount of drug-amount of drug present } \times 100}{\text { Totaldrug }}$

\subsubsection{In vitro Drug Release Studies}

The in vitro release of drug was evaluated in phosphate buffer. For this the cellophane membrane was equilibrated with simulated vaginal fluid (SVF) overnight. In vitro release studies of Amphotericin B loaded vaginal gels was carried out for 10 days at room temperature by suspending dialysis bag (prepared from cellophane membrane) containing drug loaded gel in phosphate buffer. After regular intervals of $1 \mathrm{~h}, 2 \mathrm{~h}, 3 \mathrm{~h}, 4 \mathrm{~h}$, $5 \mathrm{~h}, 6 \mathrm{~h}, 24 \mathrm{hrs}$, 1day, 2days, up to 10 days, $1 \mathrm{ml}$ of supernatant was taken out and replaced with same amount of freshly prepared phosphate buffer. The sample from supernatant was taken in a quartz cuvette and diluted with $3 \mathrm{ml}$ of distilled water. Absorbance was taken at $416 \mathrm{~nm}$ and concentration of drug was calculated from standard curve. The same procedure was followed to carry out in vitro drug release studies for Lactobacillus. Absorbance was taken at $340 \mathrm{~nm}$.

Viability of Lactobacillus from the drug released in vitro:

$100 \mu \mathrm{l}$ of phosphate buffer in which vaginal gels (containing Amphotericin B, combination of Amphotericin B with Lactobacillus) were suspended was taken at regular interval of 1 hour, 2 hours upto 6 hours and spread on trypton soya agar plates. The plates were incubated at $37^{\circ} \mathrm{C}$ for $24-48$ hours.

Bioadhesivity testing by in vitro wash off method:

The mucoadhesive properties of vaginal gels were evaluated by modified method of Patel et al. (2013) [7]. A fresh excised piece of goat intestine mucosa $(2.5 \mathrm{~cm} \times 2.5 \mathrm{~cm})$ was mounted on a glass slide. The gel sample $(1 \mathrm{~g})$ was placed over the intestine mucosa and another slide with $5 \mathrm{~g}$ of 
weight was applied over it for 5 minutes. Then the prepared specimen was transferred to the beaker placed at an angle of $60^{\circ}$ from the surface beneath. The phosphate buffer from the burette at a constant flow rate of $2 \mathrm{ml} / \mathrm{min}$ was added to the specimen. The test was carried out until the gel was removed from the mucosal surface.

For each of three vaginal gels, samples loaded with Amphotericin B, Lactobacillus alone, Amphotericin B and Lactobacillus in combination were developed to study the antimicrobial activity of the gels. Blank samples and free drug alone served as control. Supernatant of lactobacillus $100 \mu \mathrm{l}$ was added to well in a petriplates. The plates were incubated at $25^{\circ} \mathrm{C}$ for 24 hours. The study was continued for 48 hours for the blank samples so as to check the biodegradability of the crosslinked gel.

\section{RESULTS}

In the current study we aimed at developing drug delivery systems based on natural polymer carageenan encapsulating Amphotericin B and Lactobacillus against Candidal vaginitis. Bioadhesive gel systems were characterized based on $\mathrm{pH}$, swelling index and percent encapsulation.

\section{Physicochemical characterization of Carageenan gels encapsulating Amphotericin B and Lactobacillus}

pH: $\mathrm{pH}$ of vaginal gels was estimated by mixing $1 \mathrm{~g}$ of gel in $30 \mathrm{ml}$ distilled water as optimized by Ramadan (2008)[5] with the help of $\mathrm{pH}$ meter [Table 1]:

Table 1: $\mathrm{pH}$ of carageenan gels encapsulating Amphotericin B, Lactobacillus and their combinations.

\begin{tabular}{lll}
\hline S.No. & Vaginal gels & $\begin{array}{l}\text { pH } \\
\text { (Mean }+ \text { S.D) }\end{array}$ \\
\hline 1. & Carageenan - Amphotericin B & $7.2+0.10$ \\
2. & Carageenan - Lactobacillus & $6.75+0.005$ \\
3. & Carrageenan - Amphotericin B + Lactobacillus & $7.01+0.02$ \\
\hline
\end{tabular}

From above values it was seen value of $\mathrm{pH}$ increases in case of carageenan gels encapsulating drug alone. But in case of Lactobacillus values decreases.

.

Formulation And

Characterization of Carageenan Gels Encapsulating Amphotericin B And Lactobacillus acidophilus Against Candidal Vaginitis 
Kaur, R

Khurana, $\mathrm{M}$

Bindal, $\mathrm{M}$

Sharma, A
This is because of the effect of the Lactobacillus which is normal flora of vagina and tends to decrease the $\mathrm{pH}$ of the environment.

\section{Percent Encapsulation of Carageenan Gel:}

Amount of drug encapsulated in different gel systems was calculated as shown in Ramadan (2008) [5]. For this, to $1 \mathrm{~g}$ of each vaginal gel $10 \mathrm{ml}$ of methanol was added in a volumetric flask followed by continuous shaking for 10 minutes and then diluted to $100 \mathrm{ml}$ with phosphate buffer $(\mathrm{pH}=7.4)$ and centrifuged at $5000 \mathrm{rpm}$ for 10 minutes. The supernatant was filtered. The absorbance of filtrate was taken at $416 \mathrm{~nm}$ as shown in [Table 2].

Table 2: Percent encapsulation of drug (Amphotericin B) and Lactobacillus.

\begin{tabular}{lll}
\hline S.No. & Gels & $\begin{array}{l}\text { Encapsulation Efficiency } \\
\text { Mean } \pm \text { S.D }\end{array}$ \\
\hline 1. & Carageenan - Amphotericin B & $98.63 \pm 0.23$ \\
2. & Carageenan - Lactobacillus & $91.81 \pm 0.01$ \\
\hline
\end{tabular}

\section{Swelling Index:}

Swelling index is the measure of intensity of the gelation of the gel. More the swelling index, tighter the gel is. As the swelling values of vaginal gels increases, the rate of drug release also increases [Figures 1, 2 and 3].

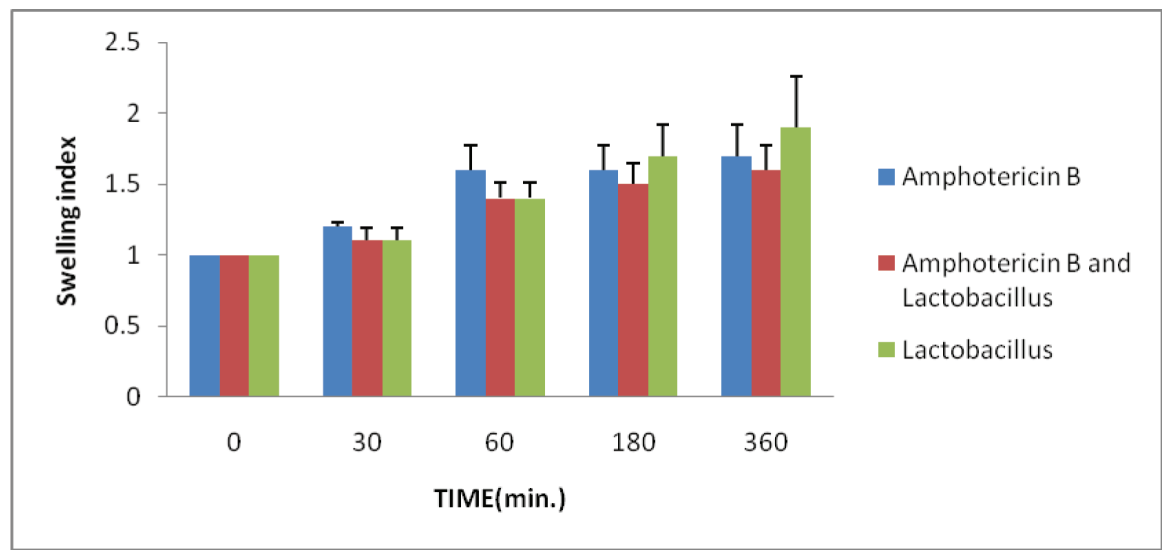

Figure 1: Swelling index of the Carageenan based vaginal gel encapsulating drug/probiotic. 


\section{Amphotericin B}

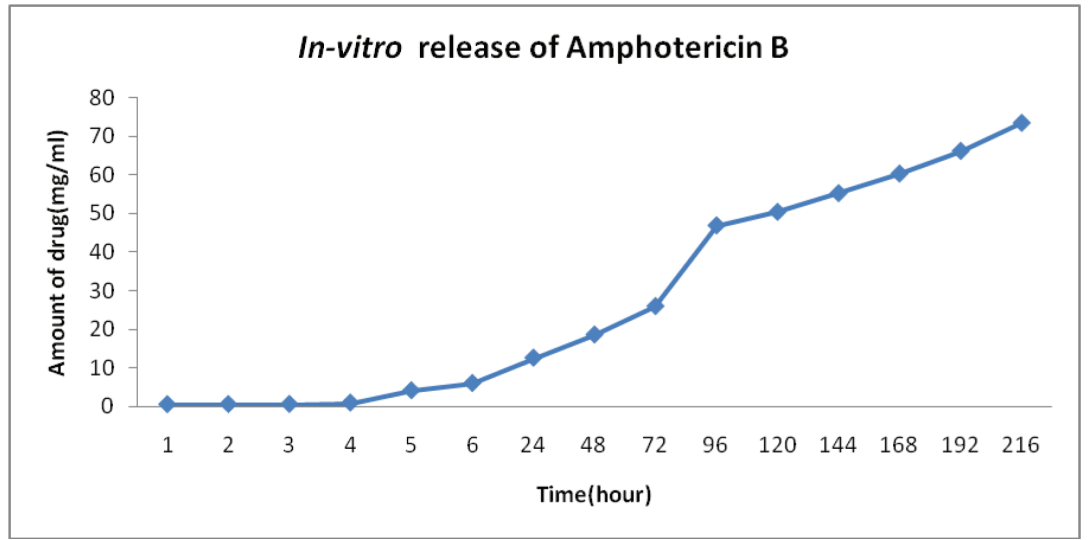

Figure 4: In vitro drug release from carrageenan gel.

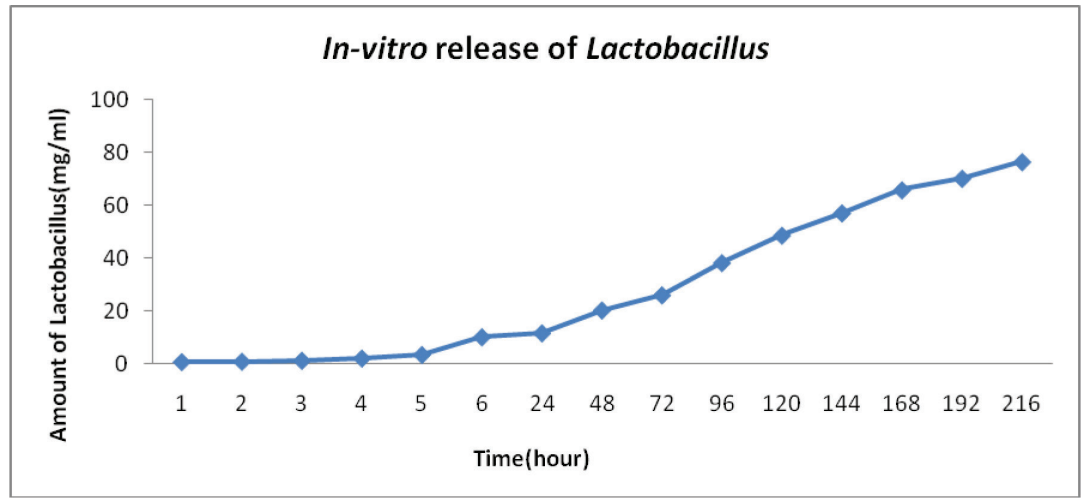

Figure 5: In vitro release of lactobacillus from carrageenan gel.

Viability of Lactobacillus in carageenan gels:

Viability of Lactobacillus was assayed in vaginal gels for 6 hours [Table 1]. $100 \mu 1$ of phosphate buffer in which vaginal gels (containing both Amphotericin $\mathrm{B}$ and Lactobacillus) were suspended, was taken at regular interval of 1 hour, 2 hours up to 6 hours and spread on trypton soya agar plates. The plates were incubated at $37^{\circ} \mathrm{C}$ for $24-48$ hours and examined.

The viability study of lactobacillus was even studied in vaginal gels as was done with gels encapsulating Carageenan gel. The viability was observed for interval of 6 hours on trypton soya agar. It was seen that viability conserved high viability till 4 hours but then it started decreasing.
Formulation And

Characterization of Carageenan

Gels Encapsulating

Amphotericin B

And Lactobacillus acidophilus Against Candidal Vaginitis 
Kaur, R Khurana, $\mathrm{M}$

Bindal, $\mathrm{M}$

Sharma, A

Table 3: Viability of Lactobacillus in carageenan gels was shown for different time intervals.

\begin{tabular}{ll}
\hline $\begin{array}{l}\text { Time Interval } \\
\text { (in hrs) }\end{array}$ & Carrageenan gel \\
\hline 1 & +++ \\
2 & ++ \\
3 & ++ \\
4 & ++ \\
5 & + \\
6 & + \\
\hline $30 \times 10^{6} \mathrm{cfu} / \mathrm{ml}$ was given '-', 30-150 $\times 10^{6} \mathrm{cfu} / \mathrm{ml}$ was given ' + ' and $>150 \times 10^{6} \mathrm{cfu} / \mathrm{ml}$ \\
was given ' ++ '.
\end{tabular}

Bioadhesive strength of vaginal gels:

Bioadhesion implies the attachment of a drug carrier to a specified location study was done to see the adherence of drugs and lactobacillus to the epithelial cells of vagina. As the bioadhesivity increases, rate of drug release also increases as shown in [Table 4].

Table 4: Bioadhesive strength of carageenan gels.

\begin{tabular}{lll}
\hline S.No. & Vaginal gel used & $\begin{array}{l}\text { Biadhesive Strength } \\
\text { Mean } \pm \text { S.D }\end{array}$ \\
\hline 1. & $\begin{array}{l}\text { Carageenan }- \\
\text { Amphotericin B }\end{array}$ & $84.66 \pm 0.5$ \\
2. & $\begin{array}{l}\text { Carageenan }- \\
\text { Lactobacillus }\end{array}$ & $88.66 \pm 0.2$ \\
\hline
\end{tabular}

\section{In vitro drug release from carageenan gel:}

The in vitro release of drug was evaluated in phosphate buffer. For this the cellophane membrane was equilibrated with simulated vaginal fluid (SVF) overnight. In vitro release studies of Amphotericin B loaded vaginal gels were carried out for 10 days at room temperature by suspending dialysis bag (prepared from cellophane membrane) containing drug loaded gel in phosphate buffer. The same procedure was followed for Lactobacillus.

Percentage of drug released up to $24 \mathrm{hrs}$ from carrageenan gel $=12.55 \%$

Percentage of drug released upto 10 days from carageenan gel $=73.38 \%$ 


\section{Lactobacillus:}

Percent of lactobacillus released upto $24 \mathrm{hrs}$ from carageenan gel $=11.41 \%$

Percent of lactobacillus released upto 10 days from carageenan gel $=78.42 \%$

\section{DISCUSSION}

Candidal vaginitis is an infection of vagina which is characterized by inflammation of female genitalia. The causative agent is yeast Candida albicans. Amphotericin B is one of the effective drugs used against candidal vaginitis. Probiotics is another strategy to use with the drugs against vaginitis as they lower the $\mathrm{pH}$ and helps in restoring the balance of microflora in vagina as demonstrated by Gil et al. (2010) [8]. There are two modes of drug delivery for the treatment of candidal vaginitis-oral and vaginal. Presently marketed formulations include oral (tablets), suppositories, vaginal rings and creams. Vaginal drug delivery presents several advantages: ease of administration, hepatic first pass-effect bypass, low systemic drug exposure and increased permeability for some drugs when comparing to the oral or the other routes. Low enzymatic activity may also benefit drug delivery through vaginal route Merabet et al. (2005) [9]. In the current study intravaginal gel systems based on bioadhesive polymers like sodium alginate, chitosan, carrageenan were developed and characterized.

Several polymers including natural (collagen, carageenan, sodium alginate, chitosan, lectin etc.) and synthetic (lactic acid based polylactic-co-glycolic acid and polyanhydride, polyorthoesters, polycynoacylates, polycaprolactone) have been employed for formulating sustained release vaginal drug delivery system. Natural polymers e.g. carrageenan, chitosan and alginate have proved better over the synthetic polymers because of better bio compatibility, non toxicity and enhanced bioavailability.

In our present study natural polymers have been choosen for sustained drug delivery system against Candidal vaginitis. Carrageenan gel was prepared by graft co-polymerization of kappa-carrageenan, using ammonium per sulphate as a free radical initiator in the presence of methylene bisacrylamide as a cross linker. This hydro gel system exhibit a high sensitivity to $\mathrm{pH}$ and serves as a platform to a wide range of pharmaceuticals. Due to sensitivity for $\mathrm{pH}$, it showed several changes of swelling and deswelling, which contributes to its suitability for controlled drug release.

The prepared bioadhesive gels showed $\mathrm{pH}$ near to the neutral $\mathrm{pH}$, which indicates that none of the formulation will cause irritation in vaginal mucosa as reported by Sudeendra et al. (2010) [10].The surface $\mathrm{pH}$ of all formulations ranged from $\mathrm{pH} 4.89$ to 7.52 as seen in [Table 1]. The vaginal gel containing
Formulation And

Characterization of Carageenan

Gels Encapsulating

Amphotericin B

And Lactobacillus acidophilus Against

Candidal Vaginitis

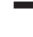


Kaur, R Khurana, $\mathrm{M}$

Bindal, $\mathrm{M}$

Sharma, A lactobacillus alone formulations shows the $\mathrm{pH}$ away from the neutral $\mathrm{pH}$ as seen in [Table 1]. Lactobacillus does not change the environment of the vagina, but it does interact with the environment to maintain normal conditions. Lactobacillus produce lactic acid, which help the vagina maintain a normal acidic $\mathrm{pH}$. It works to try and prevent any other microbes from altering its $\mathrm{pH}$ by producing a balance of lactic acid to counter any intruding microbes. It has been reported in literature that Lactobacillus bacteria are also acidophilic (acid-loving), and therefore easily tolerate the acidic $\mathrm{pH}$ of the vagina. Their optimum $\mathrm{pH}$ range for growth is from $\mathrm{pH}=3.5$ to $\mathrm{pH}=6.8$. Candida albicans grows well between $\mathrm{pH}=5.1$ and 6.9. It is important to note that Candida albicans can survive a low, acidic $\mathrm{pH}$ environment $(\mathrm{pH}=2)$, but its growth is inhibited under such acidic conditions. Hence $\mathrm{pH}$ play important role in treatment of Candidal vaginitis.

Swelling index is the measure of intensity of the gelation of the gel. More the swelling index, tighter the gel is and vice-versa. As the swelling of polymer increase, drug release rate also increase. Our study depicts the difference between the swelling index of all vaginal gels encapsulating in carageenan, chitosan and sodium alginate polymers. It was seen from graphs that swelling index values increases with time and swelling index was more in case of carageenan and chitosan gels as compared to sodium alginate gels. According to the comparison of the corresponding swelling profiles of formulations with/without lactobacillus, it c ould be s een that the lactobacillus resulted in a marked increase in swelling rate. Increased value in swelling index means gel formed is tighter and hence will result in improved drug release. Dobaria et al. (2009) have reported that the swelling capability of polymer is crucial for its bio adhesiveness as adhesion occurs shortly after the swelling ${ }^{[11]}$. Swelling is important for the assessment of adhesion. After swelling, adhesion does occur, but with a weak bond formed. The phenomenon of swelling increasing can be explained by the good disintegration effect of Lactobacillus, which made gels increase in volume and construct porous channels on surface and inside of gels. The porous channels increased the area of contacting between polymer particles and water so that the polymers could be hydrated more easily.

Percent encapsulation was observed best with Amphotericin B as compared with Lactobacillus in the vaginal gels. It depends upon interaction and encapsulation of hydrophilic drug with hydrophobic polymer. [Table 2] shows comparative percent encapsulation of Amphotericin B of carageenan, sodium alginate and chitosan gels are $98.63 \pm 0.23,98.86 \pm 0.4$ and $98.82 \pm 0.3$ respectively. It has been reported by Dhakar et al. (2012) that encapsulation efficiency depends on solubility of drug and solvent in continuous phase [12]. 
Viability of lactobacillus was observed to check the adhesion of lactobacillus to vaginal epithelial cells. It was seen [Table 3] that viability of Lactobacillus was maximum till 4 hours and decreases at 6 hour. Lactobacillus viability was highly conserved till 4 hours but then it started decreasing with time.

In vitro drug release studies were carried out in phosphate buffer under simulated vaginal conditions through a cellophane membrane. A faster release initially indicates that the drug in the solution in the space outside the gel matrix initially diffuses quickly. The release of drug within the gel is controlled by both the nature and concentration of polymer used. The bioadhesion in carrageenan may be due to the presence of more negative charges in the form of sulphate and carboxylic ions which help in attachment of gel to the mucus membrane more strongly as shown by Bhowmik and Nayak (2009) [13]. In our present study drug release kinetics of different vaginal gels showed a sustained type release of drug from the polymer i.e. there was initial burst of Amphotericin B up to 5 hours, after which there was a sustained release upto 10 days.

Bioadhesion is the formation of a bond by interfacial forces in which one surface is biological in nature. The bioadhesive strength of all vaginal gels [Table 4] results depict that vaginal gel formulation with both drug and Lactobacillus showed maximum strength.

Amphotericin B and Lactobacillus encapsulated in carrageenan gel showed bioadhesive strength $84.66 \pm 0.5$ and $88.66 \pm 0.2$ respectively. Other workers explained that Bioadhesion is a very important aspect for maintaining high drug levels at the site of administration and prevents expulsion of formulation. By maintaining effective drug concentration for longer time, one can achieve successful eradication of infectious agent.

\section{CONCLUSIONS}

Carrageenan systems were found to have maximum bioadhesive properties that help in delaying vaginal transit of encapsulated compounds. The $\mathrm{pH}$ of vaginal gels was found to be less when the Lactobacillus was encapsulated in the carageenan gel. The percent encapsulation was best with the Amphotericin B drug of vaginal gels (carageenan-98.63 \pm 0.23 ). Bioadhesivity was observed best with Carageenan gels. The viability for Lactobacillus in the gel was high till for 4 hours. Antimicrobial studies of gels encapsulating Amphotericin B and Lactobacillus showed that carrageenan gel can inhibit Candida albicans upto a maximum extent as
Formulation And

Characterization of Carageenan Gels Encapsulating Amphotericin B And Lactobacillus acidophilus Against Candidal Vaginitis

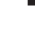


Kaur, R Khurana, $\mathrm{M}$ Bindal, $\mathrm{M}$ Sharma, A

compared to other two gels chitosan and sodium alginate. Thus it has been concluded from the present study that bioadhesive gels can act as promising therapeutic agents against candidal vaginitis.

\section{Financial support and sponsorship}

Nil

\section{Conflicts of interest}

There are no conflicts of interest

\section{REFERENCES}

[1] Donati, L., \& Di Vico, A. (2010). Vaginal microbial flora and outcome of pregnancy. Archives of Gynecology and Obstetrics, 281(4), 596-600.

[2] Gupta, A. K. (2012). Environment responsive hydrogels. Journal of drug delivery and therapeutics, 2(1), 554-557.

[3] Boskey, E. R., Cone, R. A., Whaley, K. J., \& Moench, D. R. (2001). Origins of vaginal acidity: high $\mathrm{D} / \mathrm{L}$ lactate ratio is consisitent with bacteria being the primary source. Human Reproduction, 16(9), 1809-1813.

[4] Hosseinzadeh, H. (2010). Controlled release of diclofenac sodium from pHresponsive carrageenan- g-poly (acrylic acid) superabsorbent hydrogel. Journal of Chemical Sciences, 122(4), 651-659.

[5] Ramadan, A. (2008). Formulation and evaluation of bioadhesive gels containing Miconazole Nitrate. Journal of Applied Sciences, 4(9), 1052-1065.

[6] Pal, D. K., \& Nayak, A. K. (2011). Development, Optimization, and Anti-diabetic Activity of Gliclazide-Loaded Alginate-Methyl Cellulose Mucoadhesive Microcapsules. AAPS Pharm Sci Tech, 12 (4), 1431-1441.

[7] Patel, P. V., Daslaniya, J., Patel, U. L., \& Shah, R.R. (2013). Formulation and Evaluation of Bioadhesive Gel Incorporated Amoxicillin Trihydrate Loaded Microspheres for Periodontal Therapy. International J Pharm Innovations, 3(3), 98-109.

[8] Gil, N. F., Rafael, C. R., Martinez, B., Gomes, C., Nomizo, A., Elaine, C. P., \& Martinis, D. (2010). Vaginal lactobacilli as potential probiotics against Candida spp. Brazilian J microbial, 41(1), 6-14.

[9] Merabet, J., Thompson, D., \& Saul Levinson, R. (2005). Advancing vaginal drug delivery. Expert Opin Drug Delivery, 2(4), 769-773.

[10] Sudeendra, B. R., Umme, H., Gupta, R. K., \& Shivakumar, H. G. (2010). Development and characterization of bioadhesive vaginal film of clotrimazole for vaginal candidiasis. Acta Pharmaceutica Sciencia, 52(4), 417-426.

[11] Dobaria, N. B., Badhan, A. C., \& Mashru, R. C. (2009). A novel Itraconazole Bioadhesive Film for Vaginal Delivery. American Association of Pharmaceutical Scientists, 10(3), 951-959. 
[12] Dhakar, R. C., Maurya, S. D., \& Saluja, V . (2012). From formulation variables to drug entrapment efficiency of microspheres: a technical review. Journal of Drug Delivery \& Therapeutics, 2(6), 128-133.

[13] Bhowmik, B. B., \& Nayak. (2009). Formulation and characterization of metronodazole Bioadhesive gel. International journal of pharmacy and pharmaceutical sciences, 1(17), 567-584.
Formulation And Characterization of Carageenan Gels Encapsulating Amphotericin B And Lactobacillus acidophilus Against Candidal Vaginitis 